\title{
GRAINS AND PAHS
}




\title{
INTERSTELLAR DUST GRAINS - AN OVERVIEW
}

\author{
TH. HENNING \\ Max Planck Society \\ Research Unit "Dust in Star-forming Regions" \\ Schillergäßchen 2-3, D-07745 Jena, Germany
}

\begin{abstract}
During the last years our knowledge about the structure and chemical composition of cosmic dust grains has very much deepened mainly driven by the rapid progress in infrared observations, laboratory experiments, and a much better theoretical treatment of grain evolution and the microphysical interactions of solid particles with gas, radiation, and with each other. This contribution will review the progress in the understanding of cosmic grains especially concentrating on the different grain populations, the physical processes which they link together, and the morphological and chemical structure of the particles.
\end{abstract}

\section{Introduction}

The study of cosmic dust grains is essential in understanding the dynamical, thermal, and chemical properties of the dense phases of the interstellar medium. Although the interstellar dust contributes only a minor amount to the total mass of our and other galaxies it strongly interacts with the stellar and gas components. The upper mass limit of newly formed stars and the stellar winds in distinct classes of evolved stars are directly related to the presence of solid particles (Yorke \& Henning 1994, Sedlmayr 1994). Dust opacity effects may drive or influence instabilities in protostellar flows and accretion disks (Noh et al. 1991, Duschl 1993, Lenzuni et al. 1995). In addition, dust particles are both a sink and a source for gas phase molecules and play an important role in interstellar chemistry (Wagenblast \& Williams 1993, Herbst 1993). The dust grains can carry a substantial fraction of the charge in dense, weakly ionized cosmic plasmas and, therefore, influence the electrodynamics of these regions (Havnes et al. 1987, Ciolek \& Mouschovias 1993). 
Thermal continuum emission from dust grains at infrared and submillimetre/millimetre wavelengths serves as an important indicator for the presence of cold protostars, of circumstellar disks and envelopes around young stellar objects and stars in their later evolutionary stages, and of tori around active galactic nuclei (André 1994, Beckwith 1994, Henning 1996a,b, Urry \& Padovani 1995).

There is still another aspect of cosmic dust research. The interstellar grains are a special system of nano- and microparticles (Bohren \& Huffman 1983). Therefore, many bridges to solid-state physics, chemical physics, and quantum chemistry exist. The study of novel forms of carbon was triggered by astrophysical questions (Kroto et al. 1985, Krätschmer et al. 1990, Ugarte 1995), the investigation of polycyclic aromatic hydrocarbons got a new impetus from astrophysical studies (Léger et al. 1987), and new methods for the interaction of light with irregular, anisotropic, and fluffy grains were developed by astrophysicists (Draine \& Flatau 1994, Henning et al. 1995, Stognienko et al. 1995, Michel et al. 1996).

In this review, I will discuss dust models for both the diffuse and molecular phases of the interstellar medium. Cosmic abundance constraints and new data from the analysis of primitive material in the solar system are included in the discussion. A more complete review about the dust metamorphosis in the galaxy is given by Dorschner \& Henning (1995), the properties of dust around young stellar objects are covered by Henning (1996a,b), and dust in late stages of stellar evolution is reviewed by Sedlmayr (1994).

\section{Cosmic dust - a multicomponent system}

The cosmic dust consists of several well-distinguished populations typical of the special environments in which they are formed and/or modified (Dorschner \& Henning 1995). Each population is a multi-component system containing grains or grain ingredients of different chemical composition and physical structure. There are at least four main populations:

1. Stellar outflow dust (stardust)

2. Dust in the diffuse interstellar medium (interstellar dust)

3. Dust in dense cool clouds (molecular cloud dust)

4. Circumstellar dust around young stellar objects (YSO dust).

Cool high-luminosity stars, in particular AGB and post-AGB stars, are the primary suppliers of stardust if the poorly understood process of dust formation in supernovae is not more efficient (Sedlmayr 1994, Jones \& Tielens 1994).

In the diffuse interstellar medium, grains from various sources are well mixed. Because of the frequent reprocessing of grains due to the passage of shocks, adsorption of gaseous species, irradiation by the interstellar UV field 
and cosmic rays and being in the molecular cloud phase for several periods, the interstellar dust should develop into a homogeneous sample. Destruction mechanisms connected with SN shocks are thermal and non-thermal sputtering and grain-grain collisional vaporization/shattering (Tielens et al. 1994, Jones et al. 1994, Borkowski \& Dwek 1995). The calculations by Jones et al. (1994) resulted in grain lifetimes of $4 \times 10^{8} \mathrm{yr}$ (carbonaceous grains) and $2.2 \times 10^{8} \mathrm{yr}$ (silicate grains) which are much shorter than the stardust injection timescale of $2.5 \times 10^{9} \mathrm{yr}$. Because most of the Si atoms are depleted in silicates, this would mean that an efficient grain growth process in the interstellar medium must exist and interstellar dust is not dominated by stardust injection. However, before a final conclusion can be drawn, the dust formation rate by supernovae and the grain destruction efficiency of porous grains and particles shielded by refractory mantles must be better known.

In the dense regions of molecular clouds, adsorption of gas phase species and surface chemistry leads to the formation of ice mantles. Theoretically (and partly proven by observations), one can expect mantles composed of $\mathrm{H}_{2} \mathrm{O}, \mathrm{CH}_{3} \mathrm{OH}, \mathrm{CO}, \mathrm{CO}_{2}$ and some other simple molecules with admixtures of impurities such as carbonaceous particles (Whittet 1993, 1996). Grain coagulation will modify the size distribution and leads to larger composite and fluffy grains (Ossenkopf 1993, Ossenkopf \& Henning 1994, Weidenschilling \& Ruzmaikina 1994). Similar processes but at a much higher rate are expected in the very dense protostellar cores. In protoplanetary disks including the solar nebula, the gases and grains were at least partially reprocessed by chemical reactions. In addition, dramatic changes of the grain properties due to coagulation, collisional and chemical destruction, sublimation and condensation can be expected (Morfill \& Völk 1984, Mizuno 1989, Sterzik \& Morfill 1994, Duschl et al. 1996, Schmitt et al. 1996).

\section{Grain diagnostics}

Cosmic dust grains have been studied from X-ray to radio wavelengths. Typical grain diagnostics consists of (Mathis 1993, Dorschner \& Henning 1995):

1. X-ray haloes (Predehl \& Klose 1996)

2. UV/VIS/NIR extinction and polarization curves including the absorption feature at $217.5 \mathrm{~nm}$ (Cardelli et al. 1988, 1989, Martin \& Whittet 1990, Whittet et al. 1992, Jenniskens \& Greenberg 1993, Kim \& Martin 1995)

3. Extended red emission observed in reflection nebulae, $\mathrm{H}$ II regions, and planetary nebulae and peaking between 650 and $700 \mathrm{~nm}$ (Witt \& Schild 1988, Witt \& Boroson 1990, Furton \& Witt 1992) 
4. Infrared emission and absorption features including the polarization in the features (Whittet 1993, 1996, Henning \& Stognienko 1993, Henning 1996a)

5. Infrared and (sub)millimetre thermal continuum emission (Beckwith \& Sargent 1991, Sodroski et al. 1994, Henning 1996a,b).

The diffuse interstellar bands at optical wavelengths (Herbig 1995, Tielens \& Snow 1995, Salama 1996, Freivogel et al. 1994) and the infrared emission features (Allamandola et al. 1989, Puget \& Léger 1989, Sellgren 1994) are generally not attributed to dust grains but related to C-bearing molecules (e.g. PAHs).

In the following, we will concentrate on some results of the infrared spectroscopy. The presence of the 10 and $18 \mu \mathrm{m}$ features in the spectra of a wide variety of objects (compact H II regions, Herbig Ae/Be stars, T Tauri stars, Vega-like objects, AGB stars) and along lines of sight going through the diffuse interstellar medium is generally attributed to silicates and shows their wide distribution (Little-Marenin \& Little 1988, 1990, Simpson 1991, Ossenkopf et al. 1992, Jäger et al. 1994, Dorschner et al. 1995). Evidence for other refractory components such as carbides, sulfides, and oxides is mainly based on the detection of one single band in the spectra of circumstellar envelopes around evolved stars and doubts are, therefore, reasonable (see Tab.1). The ISO spectra of dust shells around evolved oxygen-rich stars revealed the presence of several new emission features between 20 and 45 $\mu \mathrm{m}$ which are not yet reliably identified with special carriers (Waters et al. 1996).

A 3.4- $\mu \mathrm{m}$ feature has been observed in several IR sources in the Galactic Centre region, in the heavily obscured "hypergiant" Cyg OB 2 No.12, in some supergiants, and in some Wolf-Rayet stars of the types WC 9 and WC 10 (see, e.g., Pendleton et al. 1994, Sandford et al. 1995). The feature is generally attributed to $\mathrm{C}-\mathrm{H}$ stretching vibrations of saturated aliphatic hydrocarbons present in the diffuse interstellar medium. Here we should note that there is no evidence that interstellar hydrocarbon grains contain a lot of oxygen. That means that the organic grain mantles may actually have a structure quite similar to HACs which may also form by other chemical routes via the direct condensation on pre-existing cores in the diffuse interstellar medium (Duley 1993) or the modification of amorphous carbon by reactions with atomic hydrogen (Furton \& Witt 1993).

Infrared spectra of the Galactic Centre show absorption features at 3.0, 3.4, 5.5, 5.8, 6.1, 6.8, 9.7, and $19 \mu \mathrm{m}$ (Roche \& Aitken 1985, Roche 1988, Tielens et al. 1996). The 3.0 and $6.1-\mu \mathrm{m}$ features can be attributed to the presence of $\mathrm{H}_{2} \mathrm{O}$ as part of an incompletely hydrogen bonded network (Tielens et al. 1996). These features are probably produced in the molecular 


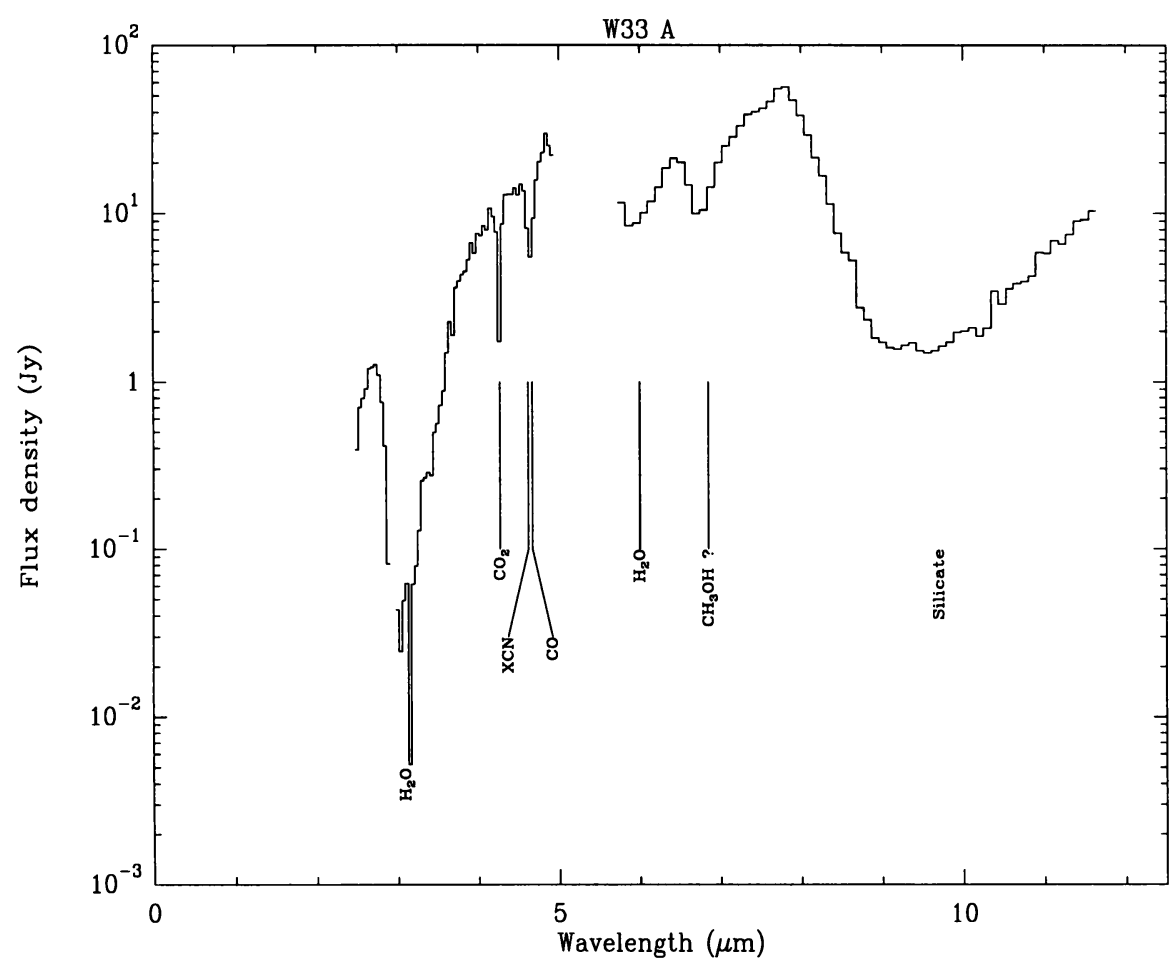

Figure 1. ISOPHOT-S spectrum of the massive young stellar object W33 A

clouds associated with the Galactic Centre sources. They are not observed along other lines of sight through the diffuse interstellar medium.

Infrared spectroscopy revealed numerous bands of cosmic "ices" between 2 and $15 \mu \mathrm{m}$ in the spectra of deeply embedded molecular cloud sources (for reviews see Schutte 1996, Whittet 1993, 1996). The most abundant mantle molecule is $\mathrm{H}_{2} \mathrm{O}$ with a pronounced feature at $3.08 \mu \mathrm{m}$. This fundamental $\mathrm{O}-\mathrm{H}$ stretch vibration has been observed in a wide variety of objects. Apart from the $3.08-\mu \mathrm{m}$ feature, the weaker $\mathrm{O}-\mathrm{H}$ bending vibrations at $6.0 \mu \mathrm{m}$ are observed. Lacy et al. (1984) detected solid CO by its stretching vibration at $4.67 \mu \mathrm{m}$ in the infrared spectra of deeply embedded and luminous YSOs. $\mathrm{CO}$ is present in both polar and non-polar matrices (see, e.g., Chiar et al. 1995). A catalogue of optical depths in the $\mathrm{CO}$ feature for a larger number of YSOs is given by Whittet \& Duley (1991). Solid $\mathrm{CO}_{2}$ was first detected by its bending vibration at $15.3 \mu \mathrm{m}$ in the IRAS LRS spectra of a few 
TABLE 1. Carbides, oxides, and sulfides in circumstellar envelopes

\begin{tabular}{llll}
\hline Wavelength & Component & Sources & References \\
\hline $11.3 \mu \mathrm{m}$ & $\mathrm{SiC}$ & Carbon-rich CSE & Baron et al. 1987 \\
\hline $13 \mu \mathrm{m}$ & $\mathrm{Al}_{2} \mathrm{O}_{3}$ & Oxygen-rich CSE & $\begin{array}{l}\text { Sloan et al. 1996, } \\
\text { Begemann et al. 1996b, } \\
\end{array}$ \\
& & & Koike et al. 1995 \\
\hline $21 \mu \mathrm{m}$ & $\mathrm{SiS}_{2}$ & $\begin{array}{l}\text { Carbon-rich CSE } \\
\text { (PPNe) }\end{array}$ & $\begin{array}{l}\text { Kwok et al. 1995, } \\
\text { Henning et al. 1996, } \\
\text { Begemann et al. 1996a }\end{array}$ \\
\hline $30 \mu \mathrm{m}$ & $\mathrm{MgS}$ & Carbon-rich CSE & $\begin{array}{l}\text { Omont et al. 1995, } \\
\text { Begemann et al. 1994 }\end{array}$ \\
\hline
\end{tabular}

embedded infrared sources (d'Hendecourt \& Jourdain de Muizon 1989). ISO observations unambiguously demonstrated the presence of the $\mathrm{C}-\mathrm{O}$ stretching vibration of the $\mathrm{CO}_{2}$ molecule at $4.27 \mu \mathrm{m}$ in quite a number of YSO spectra (Gürtler et al. 1996, de Graauw et al. 1996, Whittet et al. 1996). Another ice component which seems to be much less important is $\mathrm{CH}_{3} \mathrm{OH}$ with vibrations at $3.08 \mu \mathrm{m}(\mathrm{OH}$ stretching mode), 3.35 and 3.53 $\mu \mathrm{m}$ ( $\mathrm{CH}$ stretching modes), $6.85 \mu \mathrm{m}$ ( $\mathrm{CH}_{3}$ deformation mode), $9.75 \mu \mathrm{m}$ (CO stretching mode), and at $8.9 \mu \mathrm{m}\left(\mathrm{CH}_{3}\right.$ rocking mode). Recent results can be found in Allamandola et al. (1992) and Skinner et al. (1992). The feature observed at $6.85 \mu \mathrm{m}$ is probably a blend of different absorption components because otherwise the $\mathrm{CH}_{3} \mathrm{OH}$ abundance derived from the $6.85 \mu \mathrm{m}$ feature would be much higher and would contradict the values obtained from the $3.53 \mu \mathrm{m}$ and $9.75 \mu \mathrm{m}$ feature.

\section{Cosmic abundance constraints}

Element abundances have been widely used to constrain dust models. The fractional abundances of the elements directly lead to the main dust-forming species: oxygen, carbon, iron, silicon, and magnesium. That means that the dust grains are mainly made up of carbonaceous solids, silicates, and other iron/magnesium oxides. New determinations of gas phase abundances with the Goddard High Resolution Spectrograph aboard the Hubble Space Telescope and the NLTE analysis of B-star atmospheres point to tighter limits for the carbon and oxygen present in dust grains (Snow \& Witt 1995, 
TABLE 2. Cosmic abundance constraints

\begin{tabular}{|c|c|c|}
\hline Abundances & $\begin{array}{c}\mathrm{C} / \mathrm{H} \\
{[\mathrm{ppM}]}\end{array}$ & $\begin{array}{c}\mathrm{O} / \mathrm{H} \\
{[\mathrm{ppM}]}\end{array}$ \\
\hline $\begin{array}{l}\text { Solar } \\
\text { abundances }\end{array}$ & $\begin{array}{c}355 \pm 50 \\
\text { (Grevesse \& Noels 1993) }\end{array}$ & $\begin{array}{c}740 \pm 90 \\
\text { (Grevesse \& Noels 1993) }\end{array}$ \\
\hline $\begin{array}{c}\text { Cosmic } \\
\text { abundances } \\
\text { (young stars) }\end{array}$ & $\begin{array}{c}225 \pm 50 \\
\text { (Snow \& Witt 1995) }\end{array}$ & $\begin{array}{c}350 \pm 50 \\
(\text { Kilian et al. 1994) } \\
480 \pm 180 \\
\text { (Gies \& Lambert 1992) }\end{array}$ \\
\hline $\begin{array}{l}\text { Interstellar gas } \\
\text { abundances }\end{array}$ & $\begin{array}{c}140 \pm 20 \\
\text { (Cardelli et al. 1996) }\end{array}$ & $\begin{array}{c}310 \pm 20 \\
\text { (Sofia et al. 1994, } \\
\text { Cardelli et al. 1996) }\end{array}$ \\
\hline Cosmic grains & $\begin{array}{c}100-400 \\
\text { (different models) } \\
50 \mathrm{ppM} \text { of graphitic carbon } \\
\text { are needed to produce the } \\
217.5 \mathrm{~nm} \text { bump; } \\
140-155 \mathrm{ppM} \text { sufficient to } \\
\text { explain IS extinction including } \\
\text { the bump (Mathis } 1996 \mathrm{~b} \text { ) }\end{array}$ & $\begin{array}{c}150 \pm 30 \\
(\mathrm{Fe}, \mathrm{Si}, \mathrm{Mg} \text { in } \\
\text { completely } \\
\text { oxidized } \\
\text { state) }\end{array}$ \\
\hline
\end{tabular}

Mathis 1996a, b, Cardelli et al. 1996). The B-star abundances are much less than the solar values traditionally assumed as a reference for the interstellar medium (see Table 2). If the lower abundances are typical for the "cosmic" abundances and we take into account that about $50 \mathrm{ppM}$ of graphitic carbon are needed to produce the $217.5 \mathrm{~nm}$ bump, not much carbon is left to produce the interstellar extinction curve. Even if we use the solar value for the fractional abundance of carbon, many dust models require too much carbon (300-400 ppM) in the solids if the new gas phase $\mathrm{C} / \mathrm{H}$ values are considered. The oxygen in the dust grains could completely account for the difference between the gas phase value and the reduced reference abundance. We should note that there may be still systematic errors in the B-star abundance determinations or that the heavy elements may not be completely incorporated into stars during massive star formation. 


\section{Primitive material in the solar system}

The detection of stardust grains in primitive meteorites and interplanetary dust particles as well as new data from interplanetary spacecrafts are important sources of information concerning the nature of interstellar dust grains. The isotopic analysis led to the identification of presolar diamond, carbides, graphite, corundum, and silicon nitride grains (Anders \& Zinner 1993, Ott 1993 a,b, Zinner et al. 1995, Nittler et al. 1995). Up to now, silicate grains with a typical AGB star isotopic signature have not been detected in primitive solar system solids. However, Bradley (1994 a,b) found non-stoichiometric grains of silicate glasses with depletions of magnesium and silicon relative to oxygen and inclusions of iron-nickel metal and iron sulfides (GEMS = Glasses with Embedded Metal and Sulfide). These are strong indications for a pre-accretional origin where strong irradiation strips the cations with the weakest band strengths from the grains. GEMS are a special subgroup of polyphase grains occuring in anhydrous interplanetary dust particles. The sizes of these basic subunits range from 0.1 to $0.5 \mu \mathrm{m}$. The GEMS share many properties with interstellar silicate grains (Martin 1995 , Goodman \& Whittet 1995) although it cannot be excluded that they are early nebular condensates.

Interstellar dust grains with sufficiently low charge-to-mass ratios can penetrate the heliopause and enter the solar system. The detection of an interstellar dust component in the solar system by the Ulysses dust detector (Grün et al. 1993, 1994) opened a direct way for the investigation of such particles. The mean mass of the particles was $3 \times 10^{-13} \mathrm{~g}$ with even more massive particles present.

\section{Grain models}

Grain models for the diffuse interstellar medium are mainly based on an analysis of interstellar extinction and polarization curves taking into account cosmic abundance constraints (see Mathis 1993, Dorschner \& Henning 1995). Recent dust models are summarized in Table 3 . Here, the grain composition is given by AC (amorphous carbon), GRA (graphite), HAC (hydrogenated amorphous carbon), I (iron), PAH (polycyclic aromatic hydrocarbons), RO (refractory organics), and SIL (silicates). The size distribution is described by d (discrete size or very small size interval), exp (exponential law), g (giant grains in the order of magnitude $10 \mu \mathrm{m}$ ), $\mathrm{p}$ (power law), and vs (very small grains).

All current dust models share some common features: (1) they contain silicate and carbonaceous material, (2) they include very small grains and/or PAHs, and (3) they all claim to be able to fit the observed extinction curves. The last item is indicative of the non-uniqueness of the model 
predictions. With the non-uniqueness of the models, we mean that integral quantities (extinction) are fitted which contain both the size distribution and the optical properties of the grains. Better constraints of the models can be obtained if both the extinction and polarization curves are simultaneously modelled and spatial/temporal variations of the curves are taken into account.

The properties of the dust population in molecular cloud cores, circumstellar envelopes around YSOs, and protoplanetary disks can be considerably different from those of the dust in the diffuse interstellar medium and the envelopes around evolved stars. There are two main processes which modify the grains in the colder regions: the formation of molecular ice mantles and the coagulation of grains leading to fluffy and inhomogeneous aggregates. The optical properties of coagulated particles are a sensitive function of the assumed structure and chemical composition of the clusters (for reviews see Henning et al. 1995, Henning 1996b). In protoplanetary disks including the solar nebula, the gases and the grains were at least partially reprocessed by thermochemical reactions and shock chemistry (Prinn 1993). This may explain the wide-spread existence of FeS (troilite) in primitive bodies of the solar system. During the evolution of protoplanetary disks, dramatic changes of the opacity due to coagulation, collisional destruction, sublimation, and condensation of grains can be expected (see, e.g., Morfill \& Völk 1984, Mizuno 1989, Sterzik \& Morfill 1994, Schmitt et al. 1996).

Comprehensive dust models for protostellar cores and protoplanetary accretion disks were recently provided by Pollack et al. (1994), Henning \& Stognienko (1996), Krügel \& Siebenmorgen (1994), and Ossenkopf \& Henning (1994). The latter authors treated the dust evolution in cold molecular cloud cores together with the evolution of the optical properties of the particles self-consistently. The fluffy structure of the aggregates produced during the coagulation process was explicitly included in their calculations. The main components of this dust model are silicates, amorphous carbon, and an ice mixture. Pollack et al. (1994) considered the material composition and the element abundances in molecular cloud cores and accretion disks in detail. They are partly based on the chemical composition of primitive material found in the solar system. The authors included olivine, orthopyroxene, volatile and refractory organics, water ice, troilite, and metallic iron as major grain species. In contrast to the earlier model by Pollack et al. (1985), they did not consider hydrated silicates and magnetite. A detailed investigation of the influence of particles with a fluffy structure on the disk opacities was performed by Henning \& Stognienko (1996). A major result of this study was that the iron abundance in the different dust species plays a crucial role for the optical properties of the protoplanetary dust popula- 
TABLE 3. Dust models for the diffuse interstellar medium

\begin{tabular}{|c|c|c|c|}
\hline $\begin{array}{l}\text { Authors } \\
\text { (year) }\end{array}$ & $\begin{array}{l}\text { Grain type } \\
\text { Composition }\end{array}$ & $\begin{array}{l}\text { Size distr. } \\
\text { function }\end{array}$ & $\begin{array}{l}217.5 \mathrm{~nm} \\
\text { carrier }\end{array}$ \\
\hline $\begin{array}{l}\text { Draine and Lee } \\
(1984)\end{array}$ & $\begin{array}{l}\text { bare grains } \\
\text { SIL, GRA }\end{array}$ & $\mathrm{p}$ & GRA \\
\hline $\begin{array}{l}\text { Chlewicki and } \\
\text { Laureijs (1988) }\end{array}$ & $\begin{array}{l}\text { core-mantle + bare grains } \\
\text { core: SIL, mantle: RO, } \\
\text { bare: GRA, I, PAH }\end{array}$ & $\exp , d$ & GRA \\
\hline $\begin{array}{l}\text { Greenberg } \\
(1989)\end{array}$ & $\begin{array}{l}\text { core-mantle + bare grains } \\
\text { core: SIL, mantle: RO, } \\
\text { bare: GRA }\end{array}$ & $\exp , \mathrm{d}$ & GRA \\
\hline $\begin{array}{l}\text { Williams (1989), } \\
\text { Duley et al. (1989) }\end{array}$ & $\begin{array}{l}\text { core-mantle + bare grains } \\
\text { core, bare: SIL, } \\
\text { mantle: HAC }\end{array}$ & $\mathrm{p}, \mathrm{vs}$ & SIL \\
\hline $\begin{array}{l}\text { Mathis and } \\
\text { Whiffen (1989) }\end{array}$ & $\begin{array}{l}\text { coagulated + bare grains } \\
\text { coagulated: SIL, GRA, HAC } \\
\text { bare: GRA }\end{array}$ & p & GRA \\
\hline Wright (1989) & fractal & & GRA \\
\hline $\begin{array}{l}\text { Désert et al. } \\
(1990)\end{array}$ & $\begin{array}{l}\text { core-mantle + bare grains } \\
\text { core: SIL, mantle: RO, } \\
\text { bare: AC, PAH }\end{array}$ & $\mathrm{p}, \mathrm{vs}$ & $\begin{array}{l}\text { carbon. } \\
\text { material }\end{array}$ \\
\hline $\begin{array}{l}\text { Sorrell } \\
(1990)\end{array}$ & $\begin{array}{l}\text { bare grains } \\
\text { porous SIL, AC, GRA }\end{array}$ & d & GRA \\
\hline $\begin{array}{l}\text { Rowan-Robinson } \\
\text { (1992) }\end{array}$ & $\begin{array}{l}\text { bare grains } \\
\text { SIL, AC, GRA }\end{array}$ & $\mathrm{d}, \mathrm{g}$ & GRA \\
\hline $\begin{array}{l}\text { Siebenmorgen and } \\
\text { Krügel (1992) }\end{array}$ & $\begin{array}{l}\text { bare grains } \\
\text { SIL, AC, GRA, PAH }\end{array}$ & $\mathrm{p}$ & GRA \\
\hline $\begin{array}{l}\text { Aannestad } \\
(1995)\end{array}$ & $\begin{array}{l}\text { core-mantle + bare grains } \\
\text { core: SIL, mantle: GRA, } \\
\text { AC, RO, bare: Diamonds, } \\
\text { carbon. grains, SIL, PAH }\end{array}$ & $\mathrm{p}, \mathrm{vs}$ & GRA \\
\hline $\begin{array}{l}\text { Mathis } \\
\text { (1996b) }\end{array}$ & $\begin{array}{l}\text { coagulated + bare grains } \\
\text { coagulated: SIL, carbon, } \\
\text { oxides, bare: GRA }\end{array}$ & $p, \exp$ & GRA \\
\hline
\end{tabular}


tion. The next model step will be the self-consistent calculation of the dust evolution, optical properties, and re-coupling of the evolving opacities to the disk dynamics (Schmitt et al. 1996).

\section{References}

Aannestad, P.A. 1995, ApJ 443, 653

Allamandola, L.J., Tielens, A.G.G.M., Barker, J.R. 1989, ApJS 71, 733

Allamandola, L.J., Sandford, S.A., Tielens, A.G.G.M., Herbst, T.M. 1992, ApJ 399, 134

Anders, E., Zinner, E. 1993, Meteoritics 28, 490

André, P. 1994, in The Cold Universe, eds. Th. Montmerle, Ch.J. Lada, I.F. Mirabel, J. Trân Thanh Vân (Editions Frontières, Gif-sur-Yvette), p. 179

Baron, Y., Muizon, M. de, Papoular, R., Pégourie, B. 1987, A\&A 186, 271

Beckwith, S.V.W. 1994, in Theory of Accretion Disks-2, eds. W.J. Duschl, J. Frank, F. Meyer, E. Meyer-Hofmeister, W.M. Tscharnuter (Kluwer, Dordrecht), p. 1

Beckwith, S.V.W., Sargent, A.I. 1991, ApJ 381, 250

Begemann, B., Dorschner, J., Henning, Th., Mutschke, H., Thamm, E. 1994, ApJ 423, L71

Begemann, B., Dorschner, J., Henning, Th., Mutschke, H. 1996a, ApJ 464, L195

Begemann, B., Dorschner, J., Henning, Th., Mutschke, H., Gürtler, J., Kömpe, C., Nass R. 1996b, ApJ, in press

Bohren, C.F., Huffman, D.R. 1983, Absorption and Scattering of Light by Small Particles, (John Wiley \& Sons, New York)

Borkowski, K.J., Dwek, E. 1995, ApJ 454, 254

Bradley, J.P. 1994a, Science 265, 925

Bradley, J.P. 1994b, Geochim. Cosmochim. Acta 58, 2123

Cardelli, J.A., Clayton, G.C., Mathis, J.S. 1988, ApJ 329, L33

Cardelli, J.A., Clayton, G.C., Mathis, J.S. 1989, ApJ 345, 245

Cardelli, J.A., Meyer, D.M., Jura, M., Savage, D. 1996, ApJ 467, 334

Chiar, J.E., Adamson, J.E., Kerr, T.H., Whittet, D.C.B. 1995, ApJ 455, 234

Chlewicki, G., Laureijs, R.J. 1988, A\&A 207, L11

Ciolek, G.E., Mouschovias, T.Ch. 1993, ApJ 418, 774

de Graauw, Th. et al. 1996, A\&A 315, L345

Désert, F.-X., Boulanger, F., Puget, J.L. 1990, A\&A 237, 215

d'Hendecourt, L.B., Jourdain de Muizon, M. 1989, A\&A 223, L5

Dorschner, J., Henning, Th. 1995, Astron. Astrophys. Rev. 6, 271

Dorschner, J., Begemann, B., Henning, Th., Jäger, C., Mutschke, H. 1995, A\&A 300, 503

Draine, B.T., Lee, H.M. 1984, ApJ 285, 89

Draine, B.T., Flatau, P.J. 1994, JOSA A 11, 1491

Duley, W.W. 1993, in Dust and Chemistry in Astronomy, eds. T.J. Millar, D.A. Williams (Inst. of Phys. Publ., Bristol), p. 71

Duley, W.W., Jones, A.P., Williams, D.A. 1989, MNRAS 236, 709

Duschl, W.J. 1993, in The Chemistry of Life's Origins, eds. J.M. Greenberg, C.X. Mendoza-Gómez, V. Pironello (Kluwer, Dordrecht), p. 55

Duschl, W.J., Gail, H.-P., Tscharnuter, W.M. 1996, A\&A 312, 624

Freivogel, P., Fulara, J., Maier, J.P. 1994, ApJ 431, L151

Furton, D.G., Witt, A.N. 1992, ApJ 386, 587

Furton, D.G., Witt, A.N. 1993, ApJ 415, L51

Gies, D.R., Lambert, D.L. 1992, ApJ 387, 673

Goodman, A.A., Whittet, D.C.B. 1995, ApJ 455, L181

Greenberg, J.M. 1989, in Interstellar Dust, eds. L.J. Allamandola, A.G.G.M. Tielens (Kluwer, Dordrecht), p. 345

Grevesse, N., Noels, A. 1993, in Origin and Evolution of the Elements, eds. N. Prantzos, 
E. Vangioni-Flam, M. Cassé (Cambridge Univ. Press, Cambridge), p. 15

Grün, E., Zook, H.A., Baguhl, M., Balogh, A., Bame, S.J., Fechtig, H., Forsyth, R., Hanner, M.S., Horanyi, M., Kissel, J., Lindblad, B.A., Linkert, D., Linkert, G., Mann, I., McDonnell, J.A.M., Morfill, G.E., Phillips, J.L., Polanskey, C., Schwehm, G., Siddique, N., Staubach, P., Svestka, J., Taylor, A. 1993, Nature 326, 428

Grün, E., Gustafson, B., Mann, I., Baguhl, M., Morfill, G.E., Staubach, P., Taylor, A., Zook, H.A. 1994, A\&A 286, 915

Gürtler, J., Henning, Th., Kömpe, C., Pfau, W., Krätschmer, W., Lemke, D. 1996, A\&A 315, L189

Havnes, O., Hartquist, T.W., Pilipp, W. 1987, in Physical Processes in Interstellar Clouds, eds. G.E. Morfill, M. Scholer (Reidel, Dordrecht), p. 389

Henning, Th. 1996a, in The Cosmic Dust Connection, ed. J.M. Greenberg (Kluwer, Dordrecht), in press

Henning, Th. 1996b, in The Role of Dust in the Formation of Stars, eds. H.U. Käufl, R. Siebenmorgen (Springer-Verlag, Berlin), in press

Henning, Th., Stognienko, R. 1993, A\&A 280, 609

Henning, Th., Stognienko, R. 1996, A\&A 311, 291

Henning, Th., Michel, B., Stognienko, R. 1995, Planet. Space Sci. 43, 1333

Henning, Th., Chan, S.J., Assendorp, R. 1996, A\&A 312, 511

Herbig, G.H. 1995, Ann. Rev. Astron. Astrophys. 33, 19

Herbst, E. 1993, in Dust and Chemistry in Astronomy, eds. T.J. Millar, D.A. Williams (Inst. of Phys. Publ., Bristol), p. 183

Jäger, C., Mutschke, H., Begemann, B., Dorschner, J., Henning, Th. 1994, A\&A 292, 641

Jenniskens, P., Greenberg, J.M. 1993, A\&A 274, 439

Jones, A.P., Tielens, A.G.G.M. 1994, in The Cold Universe, eds. Th. Montmerle, Ch.J. Lada, I.F. Mirabel, J. Trân Thanh Vân (Editions Frontières, Gif-sur-Yvette), p. 35

Jones, A.P., Tielens, A.G.G.M., Hollenbach, D.J., McKee, C.F. 1994, ApJ 433, 797

Kim, S.-H., Martin, P.G. 1995, ApJ 444, 293

Kilian, J., Montenbruck, O., Nissen, P.E. 1994, A\&A 284, 437

Koike, C., Kaito, C., Yamamoto, T., Shibai, H., Kimura, S., Suto, H. 1995, Icarus 114, 203

Kroto, H.W., Heath, J.R., O'Brien, S.C., Curl, R.F., Smalley, R.E. 1985, Nature 318, 162

Krätschmer, W., Lamb, L.D., Fostiropoulos, K., Huffman, D.R. 1990, Nature 347, 354

Krügel, E., Siebenmorgen, R. 1994, A\&A 288, 929

Kwok, S., Hrivnak, B.J., Geballe, T.R. 1995, ApJ 454, 394

Lacy, J.H., Baas, F., Allamandola, J.L., Perssons, S.E., McGregor, P.J., Lonsdale, C.J., Geballe, T.R., van de Bult, C.E.P. 1984, ApJ 276, 533

Léger, A., d'Hendecourt, L.B., Bocarra, N. eds. 1987, PAHs and Astrophysics (Reidel, Dordrecht)

Lenzuni, P., Gail, H.-P., Henning, Th. 1995, ApJ 447, 848

Little-Marenin, I.R., Little, S.J. 1988, ApJ 333, 305

Little-Marenin, I.R., Little, S.J. 1990, AJ 99, 1173

Martin, P.G. 1995, ApJ 445, L63

Martin, P.G., Whittet, D.C.B. 1990, ApJ 357, 113

Mathis, J.S. 1993, Rep. Progr. Phys. 56, 605

Mathis, J.S. 1996a, in Polarimetry of the Interstellar Medium, eds. W.G. Roberge, D.C.B. Whittet (ASP Conf. Ser., San Francisco) 97

Mathis, J.S. 1996b, ApJ 472, 643

Mathis, J.S., Whiffen, G. 1989, ApJ 341, 808

Michel, B., Henning, Th., Stognienko, R., Rouleau, F. 1996, ApJ 468, 834

Mizuno, H. 1989, Icarus 80, 189

Morfill, G.E., Völk, H.J. 1984, ApJ 287, 371

Nittler, L.R., Hoppe, P., Alexander, C.M.O.D., Amari, S., Eberhardt, P., Gao, X., Lewis, R.S., Strebel, R., Walker, R.M., Zinner, E. 1995, ApJ 453, L25

Noh, H., Vishniac, E.T., Cochran, W.D. 1991, ApJ 383, 372 
Omont, A., Moseley, S.H., Cox, P., Glaccum, W., Casey, S., Forveille, T., Chan, K.-W., Szczerba, R., Loewenstein, R.F., Harvey, P.M., Kwok, S. 1995, ApJ 454, 819

Ossenkopf, V. 1993, A\&A 280, 617

Ossenkopf, V., Henning, Th. 1994, A\&A 291, 943

Ossenkopf, V., Henning, Th., Mathis, J.S. 1992, A\&A 261, 567

Ott, U. 1993a, in Protostars and Planets III, eds. E.H. Levy, J.I. Lunine (Univ. Arizona Press, Tucson), p. 883

Ott, U. 1993b, Nature 364, 25

Pendleton, Y.L., Sandford, S.A., Allamandola, L.J., Tielens, A.G.G.M., Sellgren, K. 1994, ApJ 437, 683

Pollack, J.B., McKay, C.P., Christofferson, B.M. 1985, Icarus 64, 471

Pollack, J.B., Hollenbach, D., Beckwith, St., Simonelli, D.P., Roush, T., Fong, W. 1994, ApJ 421, 615

Predehl, P., Klose, S. 1996, A\&A 306, 283

Prinn, R.G. 1993, in Protostars and Planets III, eds. E.H. Levy, J.I. Lunine (Univ. Arizona Press, Tucson), p. 1005

Puget, J.L., Léger, A. 1989, Ann. Rev. Astron. Astrophys. 27, 161

Roche, P.F. 1988, in Dust in the Universe, eds. M.E. Bailey, D.A. Williams (Cambridge Univ. Press, Cambridge), p. 415

Roche, P.F., Aitken, D.K. 1985, M.N.R.A.S. 215, 425

Rowan-Robinson, M. 1992, MNRAS 258, 787

Salama, F. 1996, in Low Temperature Molecular Spectroscopy, ed. R. Fausto (Kluwer, Dordrecht), in press.

Sandford, S.A., Pendleton, Y.J., Allamandola, L.J. 1995, ApJ 440, 697

Schmitt, W., Henning, Th., Mucha, R. 1996, in preparation

Schutte, W.A. 1996, in The Cosmic Dust Connection, ed. J.M. Greenberg (Kluwer, Dordrecht), in press

Sedlmayr, E. 1994, in Molecules in the Stellar Environment, ed. U.G. Jørgensen (SpringerVerlag, Berlin), p. 163

Sellgren, K. 1994, in The Infrared Cirrus and Diffuse Interstellar Clouds, eds. R. Cutri, W.B. Latter (ASP Conf. Ser., San Francisco) 58, p. 243

Siebenmorgen, R., Krgel, E. 1992, A\&A 259, 614

Simpson, J.P. 1991, ApJ 368, 570

Skinner, C.J., Tielens, A.G.G.M., Barlow, M.J., Justtanont, K. 1992, ApJ 399, L79

Sloan, G.C., LeVan, P.D., Little-Marenin, I.R. 1996, ApJ 463, 310

Snow, T.P., Witt, A.N. 1995, Science 270, 1455

Sofia, U.J., Cardelli, J.A., Savage, B.D. 1994, ApJ 430, 650

Sodroski, T.J., Bennett, C., Boggess, N., Dwek, E., Franz, B.A., Hauser, M.G., Kelsall, T., Moseley, S.H., Odegard, N., Silverberg, R.F., Weiland, J.L. 1994, ApJ 428, 638

Sorrell, W.F. 1990, MNRAS 243, 570

Sterzik, M.F., Morfill, G.E. 1994, Icarus 111, 536

Stognienko, R., Henning, Th., Ossenkopf, V. 1995, A\&A 296, 797

Tielens, A.G.G.M., Snow, T. eds. 1995, The Diffuse Interstellar Bands, (Kluwer, Dordrecht)

Tielens, A.G.G.M., McKee, C.F., Seab, C.C., Hollenbach, D.H. 1994, ApJ 431, 321

Tielens, A.G.G.M., Wooden, D.H., Allamandola, L.J., Bregman, J., Witteborn, F.C. 1996, ApJ 461, 210

Ugarte, D. 1995, ApJ 443, L85

Urry, C.M., Padovani, P. 1995, Publ. Astron. Soc. Pac. 107, 803

Wagenblast, R., Williams, D.A. 1993, in Dust and Chemistry in Astronomy, eds. T.J. Millar, D.A. Williams (Inst. of Phys. Publ., Bristol), p. 171

Waters, L.B.F.M., Molster, F.J., de Jong, T., et al. 1996, A\&A 315, L361

Weidenschilling, S.J., Ruzmaikina, T.V. 1994, ApJ 430, 713

Whittet, D.C.B. 1993, in Dust and Chemistry in Astronomy, eds. T.J. Millar, D.A. Williams (Inst. of Phys. Publ., Bristol), p. 9 
Whittet, D.C.B. 1996, in The Cosmic Dust Connection, ed. J.M. Greenberg (Kluwer, Dordrecht), in press

Whittet, D.C.B., Duley, W.W. 1991, Astron. Astrophys. Rev. 2, 167

Whittet, D.C.B., Martin, P.G., Hough, J.H., Rouse, M.F., Bailey, J.A., Axon, D.J. 1992, ApJ 386, 562

Whittet, D.C.B., Schutte, W.A., Tielens, A.G.G.M., et al. 1996, A\&A, 315, L357

Williams, D.A. 1989, in Interstellar Dust, eds. L.J. Allamandola, A.G.G.M. Tielens (Kluwer, Dordrecht), p. 367

Witt, A.N., Boroson, T.A. 1990, ApJ 355, 182

Witt, A.N., Schild, R.E. 1988, ApJ 325, 837

Wright, E.L. 1989, in Interstellar Dust, eds. L.J. Allamandola, A.G.G.M. Tielens (Kluwer, Dordrecht), p. 337

Yorke, H.W., Henning, Th. 1994, in Molecules in the Stellar Environment, ed. U.G. Jørgensen (Springer-Verlag, Berlin), p. 186

Zinner, E., Amari, S., Wopenka, B., Lewis, R.S. 1995, Meteoritics 30, 209

\section{Discussion}

Pecker: When one considered "spherical" grains to be typical, a lot of discussion was made about lifetimes of grains, taking into account growth factors, destruction factors (sputtering) and "expulsion" by the radiation field. Dust was found to stay a short time near hot stars, a longer time in the ISM, and finally the smaller dust grains were expelled from the galaxy by galactic radiation. The size spectrum of grains in the ISM was then rather narrow (A\&A, 1974, Pecker, and other papers). What is the present situation?

Henning: Recent models of grain sputtering in interstellar clouds indicate grain lifetimes of a few $10^{8}$ years, which are considerably shorter than the stardust injection timescale.

Williams: First, a comment: Cesare Cecchi-Pestellini and I have developed a model of dust that is consistent with the new abundance constraints. My question is: would you not agree that the materials of which dust is composed may change their nature in response to the local conditions, and so variations in the interstellar extinction curve (and other observational parameters) may be caused by such changes, in addition to possible changes in grain size distribution?

Henning: We cannot exclude this possibility at the moment. Dramatic changes of the optical properties can be expected during the growth of particles (Ossenkopf \& Henning 1994, Schmitt et al. 1996). In addition, we expect the presence of different carbon modifications depending on the hydrogen content and the radiation field of the environment. 